\title{
أثر الإستثمار السياحي في تنويع مصادر الدخل في إقليم كوردستان العراق

$$
\text { للسنوات 2003-2018 }
$$

بهرم محمود صالح" و أحمد سليمان ياسين الصفار

كلية الإدارة والاقتصاد، جامعة زاخو، اقليم كردستان - العراق.

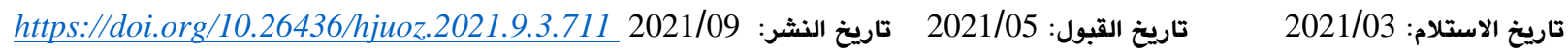

الملخص:

يحظى إقليم كوردستان العراق بثروة سياحية متنوعة وفريدة فهو مهد البشرية والمعبر الذي إستوطن فيه الجنس البشري قادما من أفريقيا ومنه إرتحل إلى

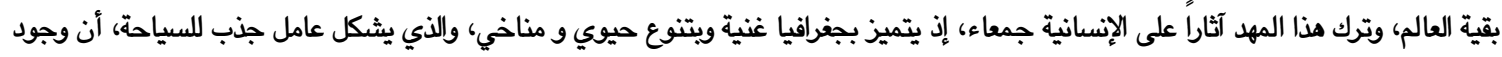

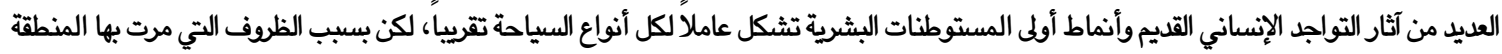
بعامة والعراق وإقليم كورستان بخاصة من حروب ومعاناة طيلة العقود الخمسة الماضية، حد من توجه الحكومة للقيام بالإهتمام بهذا القطاع، وإعتمدت القاتي

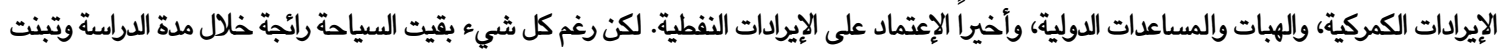

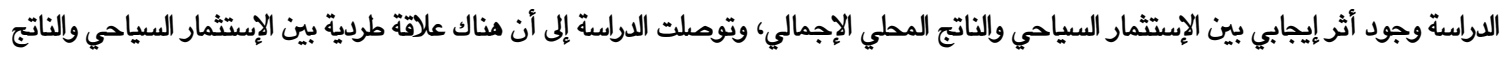

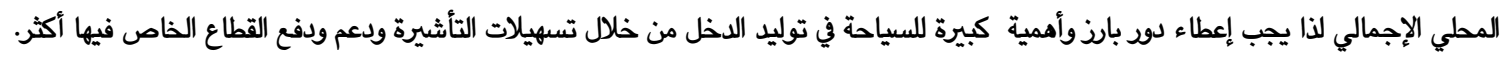
وكذلك العمل على ربط الإقليم بالعالم العربي بخطوط نقل حديثة وسريعة والمجال الجوي وفتتج أكبر عدد ممكن من الموانيء الجوية وتنمية البنى التحتية

الكلمات الدالة: الإستثمار السياحي، الإيرادات السياحية، عدد السياح، فرص العمل، الناتج المحلي الإجمالي.

رؤوس الأموال في إنشاء البنى التحتية للمنشآت والدور، والفنادق السياحية، مما

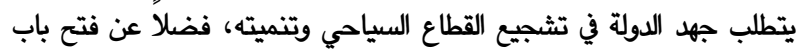
الإستثمار المحلي والأجنبي في تحقيق تلكم الأهداف.

أهمية الدراسة Importance of Study: تكمن أهمية الدراسة من أهمية القطاع السياحي في الإقليم من خلال محاولة تبني تحليل مجموعة من المتغيرات في القطاع الصيوي هذا، التي من المكن أن تهتم بها حكومة إقليم كوردستان العراق بهدف التحول إلى اقتصاد معتمد على القطاعات

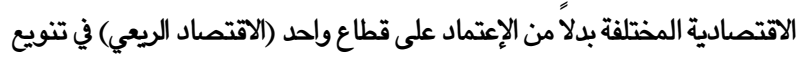
مصادر الدخل القومي، فضلاً عن إسهامه في توفير فرص العمل. أهداف الدراسة Aims of Study: تهدف الدراسة إلى تبيان أهمية الإستثمار السياحي، فضلاً عن دراسة مقومات القطاع السياحي، والعوائق التي تعتوره (تواجهه) ناهيك عن بيان أثر الإستثمار السياحي في الناتج المحلي الإجمالي في الإقليم. فرضية الدراسة Hypothesis of Study: تنص فرضية الدراسة على أن كل محّد من المحددات الثلاثة (فرص العمل، وعدد السياح، والإيرادات السياحية)، تشكل عنصراً أساسياً في تحقيق إرتفاع قيمة الناتج المحلي الإجمالي في الإقليم، ويشكل تراجع أحد المحدّات عاملاً سلبياً أمام بقية المحددات الأخرى في تحقيق إرتفاع في قيمة المؤشر المذكود آنفا.

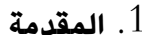

يحتل القطاع السياحي أهمية كبيرة في العديد من الدول نظراً لما له من أهمية في زيادة وتنويع مصادر الدخل القومي لأي بلد، وما تحققه هذه الزيادة من عائد مادي ومعنوي لهذه البلدان فضلا عن دوره في تنمية وتطوير البيئة والثقافة والبنية التحتية.

لا يختلف الإستثمار السياحي بالعموميات عن أنواع الإسنثمارات الأخرى، فمثلما بهتم الإستثمار بتنمية وتطوير رأس المال المادي والبشري، بهدف زيادة الإتتاج القومي كما ونوعا، فإن الإستثمار السياحي يهتم بتنمية وتطوير رأس المال المادي والبشري، الذي يعد جزءا من العملية الإنتاجية والخدمية في النشاط السياحي. يعد القطاع السياحي في إقليم كورستان العراق من أهم القطاعات الاقتصادية، وأن الإستثمار السياحي يعد عنصرا مهما من عناصر التنمية إذا ما توافرت الظروف الملائمة والذي من شأنه أنه سيسهم في تحقيق نمو إقتصادي وإجتماعي واسع من خلال إستثمار الموارد المتاحة في المناطق السياحية. مشكلة الدراسة Problem of Study: بالرغم من توافر المقومات الأساسية من تضاريس الأرض(جبال، وسهول، وهضاب، و....إلخ)، ومياه وفيرة (أنهار، وسدود، وينابيع باردة وحارة، ويحيرات، ....إخ)، وطبيعة متنوعة، وعنصر بشري، بيد أن القطاع السياحي في الإقليم

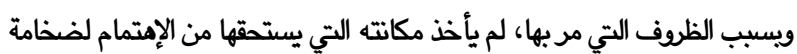


من العلاقة بين صناعة السياحة والنمو الاقتصادي بناءً على بيانات مكتب إحصاءات بلدية بكين من 1994 إلى 2015 - وإستخدمت الدراسة الناتج المحلي الإجمالي كمتغير بديل لمؤشر النمو الاقتصادي، بينما يستخدم الدخل السياحي الداخلي كمؤشر لصناعة السياحة. وتدعم الدراسة فرضية النمو بقيادة السياحة المقترحة في الأدبيات الموجودة في مختلف المسوحات للسياحة والتنمية الاقتصادية. وأظهرت النتائج أن هناك علاقة قوية في صناعة السياحة والنمو الاقتصادي في بكين، وفي الوقت نفسه، تخلق السياحة زيادة كبيرة في الإنجازات الاقتصادية الحقيقية المحلية على المدى الطويل. وتؤكد نتائج نموذج VAR أنه على المدى الطويل يتأثر النمو الاقتصادي في بكين بالسياحة المحلية، بينما يُظهر نموذج ECM نتائج أحادية الاتجاه على المدى القصير. ويالمثل هناك علاقة سببية أحادية الاتجاه بين صناعة السياحة والنمو الاقتصادي في بكين. وتدعم النتائج التجريبية بقوة مفهوم أن السياحة تسبب النمو.

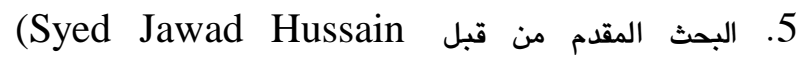
Shahzad, Muhammad Shahbaz, Roman Ferrer, and Ronald Ravinesh Kumar, 2017, p 223(232) ناقشت هذه الدراسة البحثية في الصلاحية التجريبية لفرضية النمو الذي تقوده السياحة في أفضل عشر وجهات سياحية في العالم (الصين، فرنسا، ألمانيا، إيطاليا، المكسيك، روسيا، إسبانيا، تركيا، المملكة المتحدة والولايات المتحدة) باستخدام نهج الكم على الكم وهو مؤشر جديد للنشاط السياحي الذي يجمع بين مؤشرات $(Q / Q)$

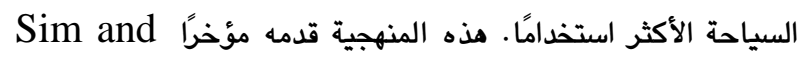

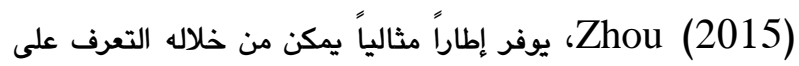
هيكل الإعتماد الكلي بين تنمية السياحة والنمو الاقتصادي. النتائج التجريبية في المقام الأول تظهر علاقة إيجابية بين السياحة والنمو الاقتصادي للبلدان العشر التي تم النظر فيها مع وجود اختلافات كبيرة بين البلدان وبين الكميات داخل كل بلد. وإن أضعف الحلقات الملحوظة هي الصين وألمانيا، ريما بسبب الأهمية المحدودة لقطاع السياحة بالنسبة للأنشطة الاقتصادية الرئيسية الأخرى في تلك البلدان. ويمكن استخلاص آثار سياسية مهمة خاصة بكل بلد من مذه النتائج. 6. ويؤكد الاقتصاديون على الخصائص الصناعية لنمو السياحة في الاقتصاد أثناء تحليل السياحة.حيث ناقش الشيل

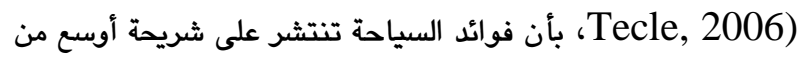
المجتمع مقارنة بقطاعات الاقتصاد الأخرى، لأن السياحة نشاط متعدد التخصصات يشمل العديد من الصناعات ويعتمد على مجموعة متنوعة من المهارات. وكشفت الدراسات الثورية لفرضية النمو الذي تقوده السياحة (Lea 1993, Sinclair 1998) عن أن النتائج المحتملة لصناعة السياحة تتمثل في خلق فرص العمل، والنمو المتطور، وتوليد
منهجية الدراسة Study Methodology: تعتمد الدراسة على المنهجين الوصفي التحليلي والكمي القائم على بناء أُنموذج قياسي إقتصادي لعينة الدراسة وللمدة (2003-2018). الدراسات السابقة Previous Studies: 1. قدمت (فاطمة مشتر وعوينان عبد القادر، 2019، ص363-372 (372 ) دراسة بحثية بعنوان الآثار التنموية لقطاع السياحة في ظل المخطط التوجيهي للتهيئة السياحية 2030. وتهدف هذه الدراسة إلى معالجة الآثار التنموية لقطاع السياحة في ظل المخطط التوجيهي للتهيئة السياحية آفاق 2030، من خلال تشخيص السياحة الدولية ومكانتها على المستوى العالمي بالإضافة إلى التطرق لتحليل كل من الطلب والعرض السياحي في الجزائر، وإبراز الدود الذي تساهم به في الإقتصاد الوطني، وعدت الدراسة أن السياحة صناعة متكاملة تتنميز بإتساع أنشطتها وتعدد أنواعها، مذا ما يميزها عن بقية القطاعات كونها نشاط إقتصادي يؤثر على الكثير من قضايا التنمية خاصة الإقتصادية منها. 2. عرضت دراسة (عليوة، 2014) تقييماً لأثر السياحة في النمو الإقتصادي في مصر وإعتمدت على نموذج الإنحار المتعدد للمتغيرات الإقتصادية المتمثلة بالناتج المحلي الإجمالي متغيراً تابعاً وإيرادات السياحة والناتج المحلي الإجمالي المتباطىء لسنة واحدة متغيرات مستقلة وقدر النموذج بالصيغة اللوغاريتمية واللوغاريتمية المزدوجة وأكدت الدراسة الإرتباط الموجب بين الناتج المحلي الإجمالي وإيرادات السياحة. وأوصت الدراسة إلى التأثير في العوامل المؤثرة في الإيرادات السياحية من خلال تحقيق الإستقراد وزيادة المشتغلين في مجال الفنادق ومتوسط تكلفة الإقامة في الغرفة السياحية من خلال زيادة تقديم الخمات الفندقية، والتركيز على زيادة الوعي الثقافي بين المواطنين بأهمية النشاط السياحي وزيادة تطوير في سلوكيات التعامل

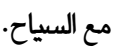
3. حلل (النادر والريموني وأرشيدات، 2010) أهم العوامل المؤثرة في تدفق الصادرات السياحية إلى الأردن وأكدت الدراسة أن معدل الدخل السياحي إلى الدخل القومي بلغ 11.2 وما يقارب 10.9٪ إلى الناتج المحلي الإجمالي، في حين بلغ معدل الدخل السياحي154 مليون دينار وعدد العاملين في هذا القطاع 27000 في عام 2007. واقترحت الدراسة بتوصية مفادها "زيادة التنسيق والتعاون بين الأردن ودول الجوار والتوجه نحو عدها إقليماً سياحياً واحداً وتنظيم حركة النقل ولديه السياحي مما يحد من المنافسة السياحية. (Yang Songling, Muhammed Ishtiaq, 4راسة and Thi thanh, 2019, p 1-14) السياحة والنمو الإقتصادي في بكين الصين. خلصت إلى أنه في الاقتصاد النامي ، تعتبر السياحة الواجهة الأكثر وضوحا وثباتا ، وتعد السياحة أحد العناصر المسرعة للتنمية الاقتصادية خلال العقدين الماضيين. لذلك استخدمت الدراسة المقترحة نموذج الانحدار الذاتي المتجه ونموذج تصحيح الخطأ (VAR) 
وخصوصاً في المدة الأخيرة المتمثلة بتطور وسائل الإتصال والمواصلات، إذ تقاربت البلدان المختلفة وزادت شدة الإتصال

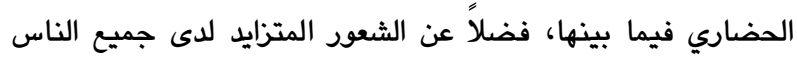

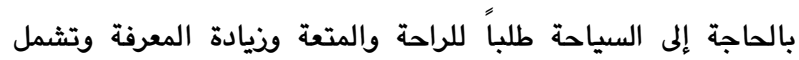
السياحة عدداً من الأنشطة (السياحة الدينية والحضارية والثقافية

والصحية والترفيهية وغيرها).

\section{2. أهمية الإستثمار السياحي:} يعد الإستثمار من أهم الموضوعات التي حظيت بإهتمام الإقتصاديين والسياسيين، نظراً لما له من أثر فعال وعلاقة وثيقة في زيادة الدخل القومي وتحقيق عملية التنمية الإقتصادية والإجتماعية إذ أن أي زيادة في الإستثمار تؤدي إلى زيادة مضاعفة في الدخل والإنتاج والإستخدام

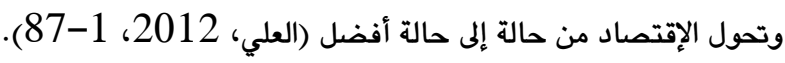
ويعد التطور الكبير الذي شهدته السياحة وإتساع حجمها كنشاط فاعل

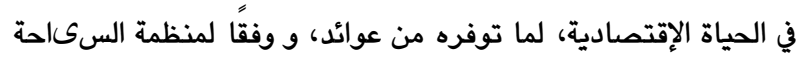

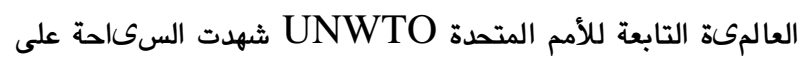
مدى العقود الستة الماضىة نمواً وتنوعا مستمرىن لتصبح السىاحة صناعة رىئسى مالى عالمىا وقطاع رئىسي في العدىد من الاقتصادات. و واحدة من أكبر القطاعات الاقتصادىة وأسرعها نمواً في العالم. بمرود الوقت، انفتح عدد متزاىد من الإستثمارات في تطوير

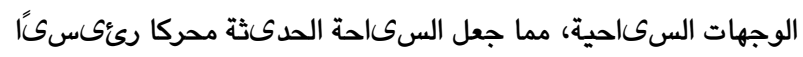
للتقدم الاجتماعي والاقتصادي. وتؤكد منظمة السىاحة العالمىة على أن السىاحة أصبحت واحدة من فئات التجارة الدولىة الرئىسىة. عحتل دخل الصادرات الذي تحققه السىاحة

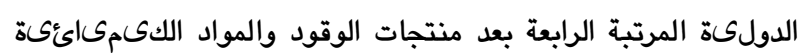

$$
\text { والسىارات. }
$$

وياعتبار السياحة واحدة من أكبر القطاعات الاقتصادية في العالم ، تعمل السياحة والسفر على خلق الوظائف، ودفع عجلة التصدير، وتوليد الرخاء في جميع أنحاء العالم. في تحليل المجلس العالمي للسياحة والسفر السنوي للأثر الاقتصادي العالمي للسياحة والسفر، ظهر أن القطاع يمثل 10.4 ٪ من الناتج المحلي الإجمالي العالمي و 313 مليون وظيفة، أو 9.9 ٪ من إجمالي العمالة، في عام 2017

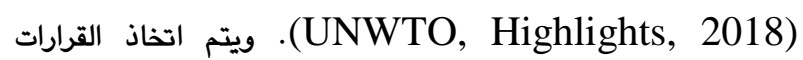
السياسية والاستثمارية الصحيحة فقط مع الأدلة التجريبية. منذ أكثر من 25 عامًا، يقدم المجلس العالمي للسياحة والسفر (WTC) مذه الأدلة لتحديد كمية الأثر الاقتصادي والتشغيلي للسياحة والسفر. وتغطي تقارير المجلس الاقتصادية السنوية لعام 2018185 دولة و 25 منطقة في العالم، وتوفر البيانات اللازمة عن أداء عام 2017 بالإضافة إلى تنبؤات فريدة مدتها 10 سنوات حول إمكانات القطاع.
الإيرادات للحكومة. وعلى أساس هذه الفرضية، ذكر سنكلير وستابلر (2002) إعتبار السياحة الدولية العامل الاستراتيجي المحتمل للنمو الاقتصادي، لأنها توفر النقد الأجنبي والإنفاق السياحي وشكل بديل للصادرات. أما الدراسة الحالية سوف تعتمد أسلوب القياس الإقتصادي لبيان أثر الإستثمار السياحي المتمثل في (الإيرادات السياحية، وعدد السياح، وفرص العمل) كمتغيرات مفسرة في تنويع مصادر الدخل في إقليم كوردستان العراق للسنوات 2003-2018. والمتمثل في الناتج المحلي الإجمالي كمتغير معتمد.

\section{2. مفهوم الإستثمار السياحي وأهميته}

$$
\text { 1.2. مفهوم الإستثمار السياحي: }
$$

يعرف الإستثمار السياحي بأنه ذلك الجزء من الدخل الذي لا يستهلك

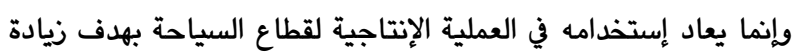
توفير الخدمات السياحية أو المحافظة عليها ويدخل في ذلك الإضافة إلى المخزون السلعي والخدمي لهذا القطاع. ويعد الإستثمار الوجه الثاني من أوجه الفعاليات الإقتصادية الرئيسة في الإقتصاد (عريقات، 2006، (109

ويعرف بأنه عبارة عن الإضافة إلى تكوين رأس المال العيني المتمثل في زيادة الطاقة الإنتاجية والمحافظة على الطاقات الإنتاجية القائمة وتجديدها، أي أن الإستثمار بهذا المفهوم يمثل إضافة إلى رأس مال

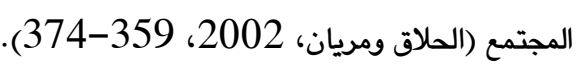
ويعرف أيضاً بأنه توظيف الأموال المتاحة في أصول رأسمالية متنوعة للحصول على تدفقات مالية أكثر في المستقبل (آل شبيب، 2009،

وتعرف السياحة بأنها الصناعة العالمية المتكاملة للسفر والإقامة والمواصلات وسائر المكونات الأخرى شاملة للتأسيس والدعم، والتي تخدم وتشبع إحتياجات ورغبات المسافرين، كما تعرف أيضاً بأنها مجموعة من الأنشطة والخدمات والصناعات التي تتكون منها خبرة السفر، والنقل والمواصلات ومؤسسات الطعام والشراب والمحلات

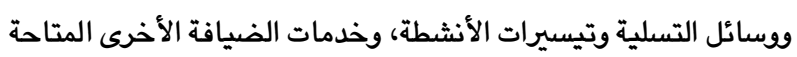

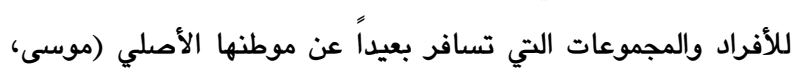
2013، 192). ويشير ذلك إلى عد صناعة السياحة منظومة متكاملة من العلاقات التي تمتاج إلى رؤية سياسية وإقتصادية وإجتماعية وقانونية تسهم في تفعيل هذا القطاع الحيوي والمهم وإبراز دوده في زيادة

$$
\text { مصادر الدخل (عيسى وحسن، 2016، 1-26). }
$$
وتعد السياحة ظاهرة إقتصادية وإجتماعية وحضارية تطورت عبر الزمن، وإزدادت أهميتها عبر نتيجة التطور العلمي والتقني الحديث واهيث 
والثقافية والبيئية، إذ يعود الإستثمار في القطاع السياحي بالعديد من الفوائد والمنافع الإقتصادية للدولة والمجتمع ومن هذه المنافع الإقتصادية هو المساهمة في زيادة الناتج المحلي الإجمالي بما ينتجه القطاع السياحي من سلع وخدمات، فضلاً عن تدفق رؤوس الأموال

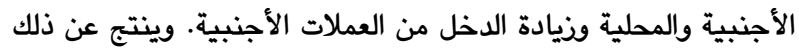
تحسن في ميزان المدفوعات، وزيادة في القيمة المضافة وتنويع مصادر

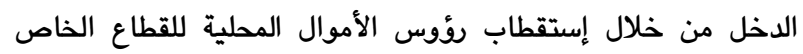
وإستثمارها في مجال الحرف والصناعات التقليدية ذات العلاقة بصناعة

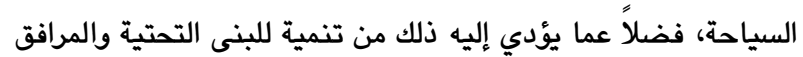

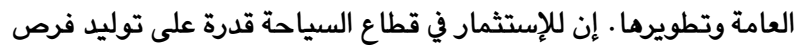
عمل جديدة، وعلى الأخص في القطاع الذدمي بشكل يفوق النشاطات

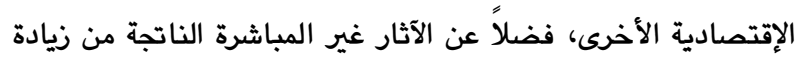
فرص العمل والحد من البطالة وإنخفاض نسبتها، كما يترتب على زيادة فرص العمل إرتفاع مستوى الرفاهية الإجتماعية وغير ذلك من المنافع

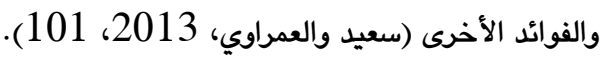

\section{3. مقومات وعوائق الإستثمار السياحي في إقليم كوردستان العراق}

1.3. مقومات الإستثمار السياحي في إقليم كوردستان العراق: يتميز القطاع السياحي بأهميته الكبيرة ودوره الفاعل في دعم الإقتصاد

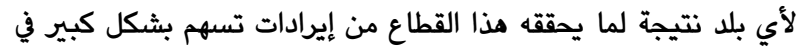
دعم عملية التنمية، ولقد عانى القطاع السياحي في الإقليم من تدهود

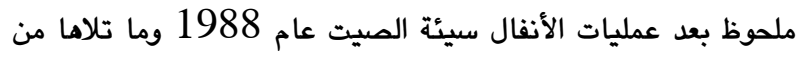

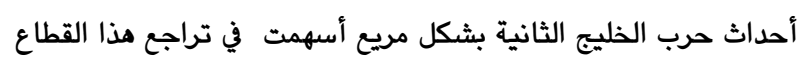

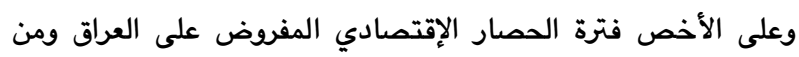

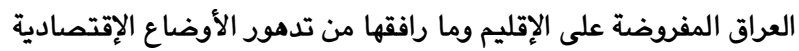
والسياسية والأمنية والثقافية والإجتماعية والسياحية حتى عام 2003 عام تغير الحكم في العراق وتعرض الكثير منها للسلب والنهب والدمار.

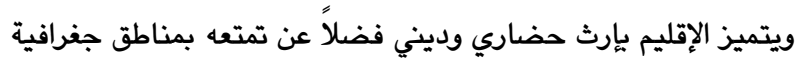
ذات مناخ معتدل ومتنوع، وتتمثل أبرز مقومات وإمكانات السياحة في

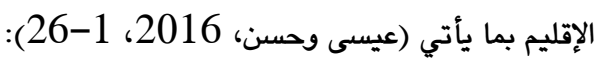
1- الإمكانات الطبيعية: تعد الإمكانات الطبيعية من أهم المقومات التي تدعم وتحفز ظهور السياحة وتطورها في أي بلد وتتمثل بالموارد المائية والحيوانية والنباتية فضلاً عن المعالم الجغرافية التي توفر المناخ السياحي، ويمتلك إقليم كوردستان العراق العديد من الإمكانات الطبيعية والتي تتميز بها مختلف مناطقه فوجود الجبال والوديان والشلالات المائية وإعتدال المناخ فيه جعل منه أمم مورد طبيعي سياحي فريد من نوعه والتي ييميز بروعة المناظر ووجود ثروات حيوانية برية فضلاً

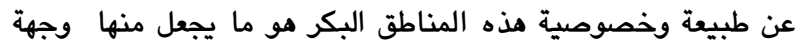

حيث كان عام 2017 واحدة من أقوى سنوات نمو الناتج المحلي الإجمالي في عقد من الزمان مع إنفاق المستهلكين القوي في جميع أنحاء

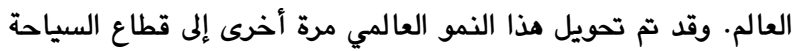
والسفر بنسبة نمو للقطاع تقدر بـ

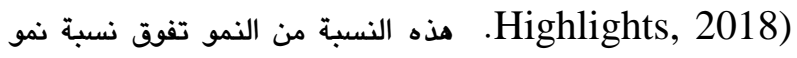
الاقتصاد العالمي للسنة السابعة على التوالي.

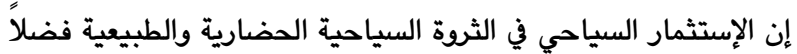
عن الإستثمار في مجال الخدمات والتسهيلات السياحية ستكون له منافع إقتصادية وإجتماعية وثقافية وبيئية على النطاق المحلي، وكما أشرنا

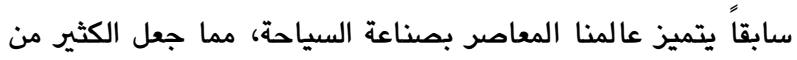
البلدان تهتم بالإستثمار السياحي نظراً لمزاياه الإيجابية المتعددة في المجالات الاقتصادية والإجتماعية والثقافية والبيئية، وقد أصبحت السياحة ذات أثر فاعل في دعم الاقتصاد العالمي وتنشيط حركة

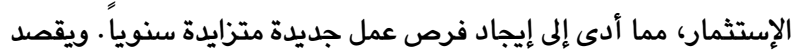

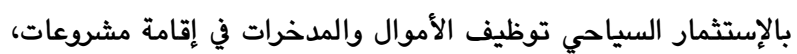
عن طريق توظيف المدخرات في شراء أو إنتاج معدات وآلات وأجهزة ومبان، أي إستخدام تلك المدخرات في زيادة الطاقة الإنتاجية، إذ تعمل الطاقة الإنتاجية على توفير المزيد من الذمات لأفراد المجتمع والدولة

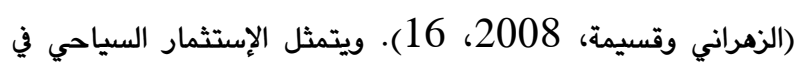
اقتصادات السياحة بتقييم المشروعات أو دراسات الجدوى الاقتصادية للمشروعات من حيث التوقعات لكل من النفقات والإيرادات، وتقدير الأرباح المتوقعة أو معدل العائد السنوي على الأموال المستثمرة ثم مقارنتها بسعر الفائدة السائد. كما يمكن تعريف الإستثمار السياحي

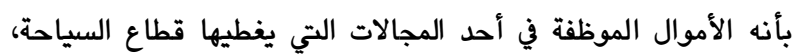
وتشمل الإستثمار في المقومات والإمكانات الرئيسة لصناعة السياحة التي يمكن إجمالها في محورين رئيسيين هما (الزهراني وقسيمة، 2008

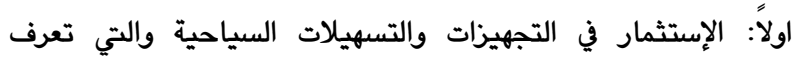

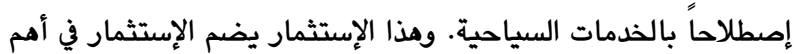
ثلاثة قطاعات خدمية هي: 1 ـ خدمات الإقامة والإعاشة والتسهيلات الترفيهية. 2. خدمات النقل والبنية التحتية.

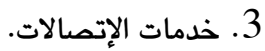

ثانياً: الإستثمار في مجال الثروة السياحية ويتمركز الإستثمار في هذا المجال بصورة رئيسة في مواقع الجذب السياحي وموارده المتمثلة في:

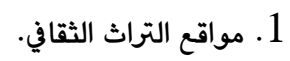
2. مواقع التراث الطبيعي. ويهدف الإستثمار السياحي في الموارد الثقافية الطبيعية والتجهيزات والخدمات السياحية إلى تحقيق عدد من المنافع الإقتصادية والإجتماعية 
5- عدم توافر برامج سياحية متميزة تسهم في إطالة إقامة السياح في الإقليم ومن شأن ذلك أن يؤدي إلى إضعاف الخدمات السياحية المقدمة. 6- 6دم توافر بيانات ومعلومات كافية بسبب غياب النظام الجيد

$$
\text { للمعلومات والإحصاء السياحي. }
$$
وعليه فإن القطاع السياحي في إقليم كوردستان العراق يواجه تحديات كبيرة تتمثل في إختفاء وتهديد قلعة أرمشت ويقايا مدينة زعفران في قصبة زاخو والسليفاني وكهوف خنس الأثرية قضلاً عن التهديد الأمني المستمر للمواقع السياحية الدينية وضياع وفقدان العديد من القطع

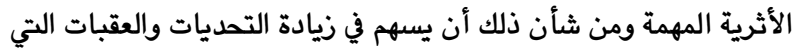
تواجه القطاع السياحي في الإقليم وبالتالي عدم القيام بدوده في ظلل التحديات التي تواجه إقتصاد الإقليم والمتمثلة بنقص التمويل

$$
\text { وإنخفاض واردات القطاع النفطي. }
$$

4. واقع السياحة في إقتصاد إقليم كوردستان العراق للمدة (2018-2003)

\section{4. تطور الطلب السياحي في الإقليم:}

شهدت أعداد السياح زيادة بعد عام 2003 نتيجة الزيادة في مستويات الدخول وتخفيف أثر الأزمة الحمار العالمي، مما دفع إلى إرتفاع

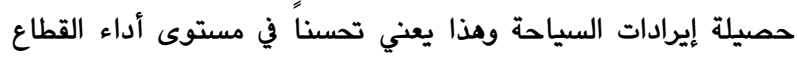
السياحي، حيث بدأت عملية الإعمار ويناء الفنادق والمنتجعات السياحية، بل أن بعض الفنادق في محافظات أربيل والسليمانية ودهوك تخضع للتصنيف الفندقي العالمي وكلها تعمل بسبب إرتفاع الطلب

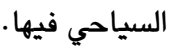
ويبين الجدول (1) عدد السياح والأهمية النسبية وحصة الإقليم من السوق السياحية العالمية و الذي شهد تزايداً منذ عام 2003 إلى على

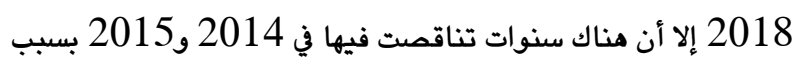
الحرب ضد الإرهاب والإضطرابات التي حدثت مما أثر في نقص عدد السياح والإيرادات. ويدأت الزيادة في عدد السياح من عام الإمات التيات 2003 وصولاً إلى عام 2013 حيث وصل عدد السياح في الإقليم ما يقارب 2.9 مليون زائر إلا أن عام 2014 وما تلاه من الظروف التي مر بها العراق ويالأخص إقليم كوردستان من ظروف الحرب ضد الإرهاب أدى إلى فوضى وعدم إستقرار أمني كبيرين مما قلل من عدد السياح

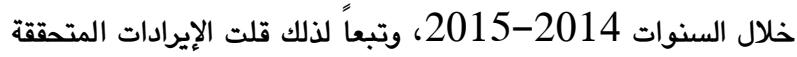
من هذا القطاع فبعد أن أصبحت 366 مليون دولار عام 2013 إنففضت إلى 101 مليون دلار عام 2015 مما قلل من مساهمة هذا القطاع في تنويع مصادر الدخل بسبب ظروف الحرب من جهة وعدم الإستقرار الأمني الذي شهده المنطقة بأسرها.
سياحية مهمة وعلى الأخص مع توافر عنصر الأمان في الإقليم، ويشير ذلك إلى ثقل الإقليم وسعته والذي أصبح محط أنظار العديد من السياح المحليون والأجانب على حد سواء مما يبشر بمستقبل واعد لقطاع السياحة في الإقليم والذي يتضمن العديد من المصايق والمناطق السياحية (شلالات بيخال، مصيف صلاح الدين، مصيف سرةرش، سهل حرير، كهف شانيدر، مضيق وشلال كلي علي بك، سرسنك، قلعة

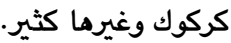
2- الإمكانات والمقومات التاريخية والحضارية: يعد إقليم كوردستان

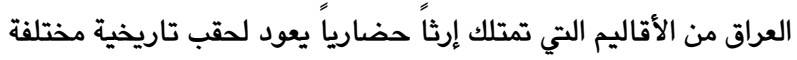

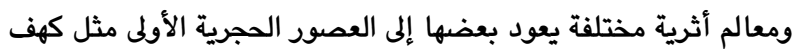
شانيدر وقرية جرمو في جمجمال في كركوك وعدد من الأماكن تعود للحضارة الآشورية وغيرها الكثير في مختلف المناطق، مما يجعل من الإقليم مركزاً للإرث الحضاري العالمي. 3- الإمكانات والمقومات الدينية: يمتلك الإقليم العديد من المعالم الدينية في مختلف مناطقه والتي تعود للعديد من الديانات السماوية فهناك دود العبادة والجوامع والمراقد والكنائس وقبور الأنبياء ومن

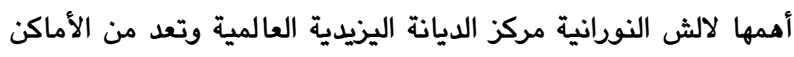

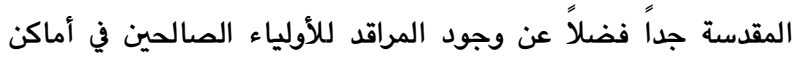
مختلفة من الإقليم وغيرها كثير. مما تقدم يمكن القول بأن السسياحة الدينية في الإقليم لها خصوصيتها، ونتيجة لذلك فهي تشكل أحد

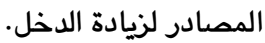

2.3. عوائق الإستثمار السياحي في إقليم كوردستان العراق: تتوافر الإمكانات والمقومات لقيام السياحة في الإقليم ونموها، إلا أنها

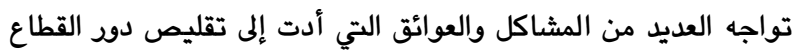

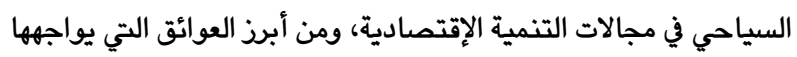

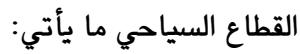
1- الإفتقار إلى ستراتيجية للإقليم واضحة المعالم حول السياحة وآفاق تطورها بما يحدد الطريق نحو تحقيق التطور في هذا القطاع ويما يتلائم مع حجم الإمكانيات المتاحة. 2- عدم وجود بنية تحتية مناسبة تتلائم مع حجم هذه الإمكانيات وضعف الخدمات وقصور في المرافق الأساسية كالطرق والكهرباء والإتصالات والصرف الصحي. 3- عدم توافر وعي إجتماعي بأهمية القطاع السياحي ودوده في التنمية الإقتصادية والبيئية والإجتماعية، وإممال المناطق الأثرية والطبيعية من خلال التقصير في أعمال الصيانة والترميم وإعادة البناء وإجراء المزيد

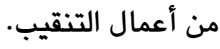
4- التهريب الذي يطول الآثار و القطع الأثرية وعدم متابعته والعمل على إرجاع ما سرق منها مما أثر سلباً في القطاع السياحي. 
سنوي متزايد بلغ (14.41) وهو معدل نمو مرتفع وهذا يتطلب الإمتمام بهذا القطاع فهو مصدر متجدد مستدام يمكن الإستفادة منه مصنه بمساهمته في توليد الدخل القومي الكوردستاني.
لكن على الرغم من كل تلك الظروف إلا أن القطاع السياحي شهد إنتعاشاً

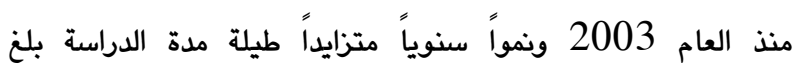
(26.07٪)، وكما أن الإيرادات السياحية المتحققة حققت معدل نمو

\begin{tabular}{|c|c|c|c|c|}
\hline حصة الإقليم من & الأهمية & معدل التغيير (\%) & عدد السياح & السنة \\
\hline 0.00008 & $0.27 \%$ & - & 54632 & 2003 \\
\hline 0.00013 & $0.48 \%$ & 77.10 & 96751 & 2004 \\
\hline 0.00018 & $0.73 \%$ & 50.67 & 145780 & 2005 \\
\hline 0.00031 & $1.33 \%$ & 82.11 & 265489 & 2006 \\
\hline 0.00038 & $1.89 \%$ & 42.21 & 377397 & 2007 \\
\hline 0.00034 & $2.80 \%$ & 48.08 & 558860 & 2008 \\
\hline 0.00049 & $3.96 \%$ & 41.60 & 791345 & 2009 \\
\hline 0.00079 & $6.58 \%$ & 66.03 & 1313841 & 2010 \\
\hline 0.00100 & $8.53 \%$ & 29.57 & 1702390 & 2011 \\
\hline 0.00124 & $11.11 \%$ & 30.23 & 2216993 & 2012 \\
\hline 0.00159 & $14.79 \%$ & 33.15 & 2952027 & 2013 \\
\hline 0.00080 & $7.66 \%$ & -48.19 & 1529434 & 2014 \\
\hline 0.00040 & $3.92 \%$ & -48.85 & 782251 & 2015 \\
\hline 0.00079 & $8.03 \%$ & 104.97 & 1603400 & 2016 \\
\hline 0.00117 & $12.54 \%$ & 56.02 & 2501678 & 2017 \\
\hline \multirow[t]{2}{*}{0.00138} & $15.32 \%$ & 22.20 & 3057000 & 2018 \\
\hline & $100 \%$ & & 19949268 & المجموع \\
\hline
\end{tabular}

المصدر: العمود الثاني من الهيئة العامة للسياحة أربيل، والأعمدة من الثالث إلى الخامس من عمل الباحث.

الخدمات فضلاً عن الأثر المضاعف للدخل الذي يولده الإنفاق من دوران الإيرادات السياحية في دورات إقتصادية متنوعة. ويوضح الجدول (2) مدى مساممة الإيرادات السياحية في تكوين الدخل القومي المتمثل

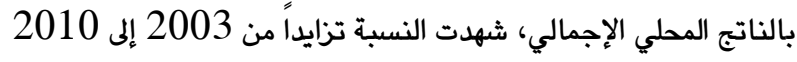
من ثم شهدت ضعفاً في مساهمة الإيرادات السياحية كنتيجة لبدأ عملية تصدير النفط من حقول كوردستان وإزدياد الإيرادات النفطية
2.4. واقع مساهمة السياحة في إقتصاد الإقليم:

إن مساهمة قطاع السياحة من خلال دوره في توفير العملة الأجنبية وفي توفير فرص العمل وكذلك تحقيق فائض في الميزان السياحي الذي يسهم في تحقيق فائض في الميزات التجاري من خلال بعض المؤشرات:

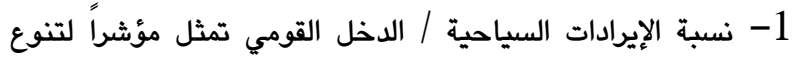

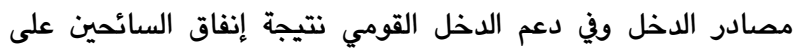

\begin{tabular}{|c|c|c|c|}
\hline الإيرادات/الناتج المحلي & الإيرادات السياحية (مليون & $\begin{array}{c}\text { الناتج المحلي الإجمالي } \\
\text { (مليون دولار) }\end{array}$ & السنوات \\
\hline 0.007 & 16.3 & 2068 & 2003 \\
\hline 0.005 & 25.6 & 4695 & 2004 \\
\hline 0.006 & 39.2 & 6404 & 2005 \\
\hline 0.011 & 85.2 & 7536 & 2006 \\
\hline 0.012 & 117.4 & 9871 & 2007 \\
\hline 0.016 & 194.5 & 12374 & 2008 \\
\hline 0.031 & 320.5 & 10498 & 2009 \\
\hline 0.035 & 456 & 13023 & 2010 \\
\hline 0.020 & 410.3 & 20513 & 2011 \\
\hline 0.012 & 277.1 & 23590 & 2012 \\
\hline
\end{tabular}


صالح ، بهرم محمود و الصفار، أحمد سليمان ياسين / مجلة العلوم الانسانية لجامعة زاخو، مجلد:9 ، العدد:3 ، ص 621- 632 ئيلون- 2021.

\begin{tabular}{c|c|c|c}
\hline 0.020 & 366.1 & 18050 & 2013 \\
\hline 0.044 & 267.7 & 6016 & 2014 \\
\hline 0.022 & 101 & 4551 & 2015 \\
\hline 0.041 & 182.8 & 4484 & 2016 \\
\hline 0.042 & 280.2 & 6682 & 2017 \\
\hline 0.043 & 327.1 & 7665 & 2018 \\
\hline
\end{tabular}

المصدر: وزارة التخطيط، الخطة التنموية للسنوات 2015-2019. نسبة معقولة جداً من العاملين وأن معدل نمو المشتغلين في السياحة

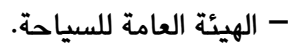

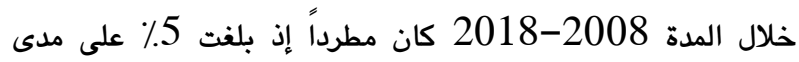

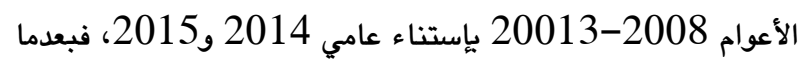
كانت فرص العمل الإجمالية نحو22 الف فرصة عمل عام 2008،

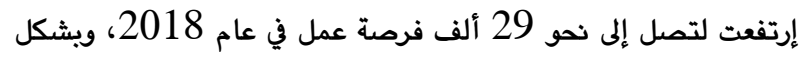

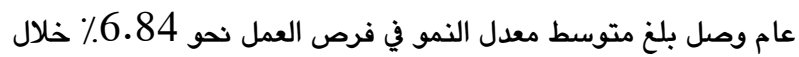
المدة 2008-2018.(الهيئة العامة للسياحة، 2018، أربيل). ويوضح الجدول (3) فرص العمل التي يوفرها القطاع السياحي في الئه

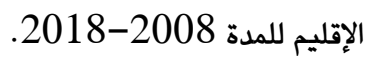

- البنك المركزي العراقي: المديرية العامة للإحصاء والأبحاث، النشرة الإحصائية السنوية، أعداد مختلفة- وموقع البنك الدولي، متوفر على لئ لهرئ الرابط: http://data.albankaldawli.org/indicator/NY.GD $\underline{\text { P.MKTP.CD }}$

لتطغي على مساهمة الإيرادات السياحية، لكن نلاحظ بأنه وعند هبوط أسعار النفط في منتصف 2014 ووصولها إلى 17 دلار رجعت نسبة المساممة في الإزدياد لتصل إلى 4\% على الرغم من أن هذا المصدر يعد خامس مصدر أساسي في تكوين الدخل في بعض الدول. 2- توفير فرص عمل: تساهم السياحة في توفير فرص العمل في الإقتصاد مما يعمل على تخفيض نسبة البطالة وأن هذا القطاع فيه

\begin{tabular}{|c|c|}
\hline فرص العمل & السنة \\
\hline 17506 & 2003 \\
\hline 18427 & 2004 \\
\hline 19397 & 2005 \\
\hline 20418 & 2006 \\
\hline 21493 & 2007 \\
\hline 22624 & 2008 \\
\hline 23815 & 2009 \\
\hline 25068 & 2010 \\
\hline 26387 & 2011 \\
\hline 27776 & 2012 \\
\hline 29238 & 2013 \\
\hline 15777 & 2014 \\
\hline 12397 & 2015 \\
\hline 23413 & 2016 \\
\hline 29281 & 2017 \\
\hline 30173 & 2018 \\
\hline
\end{tabular}

المصدر: - الهيئة العامة للسياحة، 2018، وزارة البلديات والمصايف، أربيل. من خلال وضع ستراتيجية وطنية إقتصادية لهذا القطاع تأخذ بنظر - الهيئة العامة للإحصاء، 2018، وذارة التخطيط، أربيل.

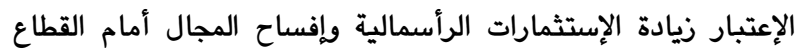

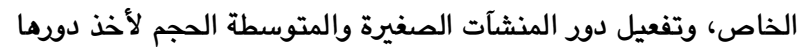
إن مسألة توفير وزيادة فرص العمل في إقليم كوردستان العراق سيبقى

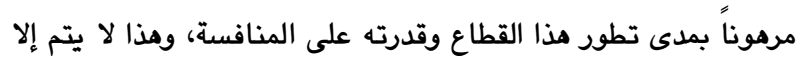


أنها ذات تأثير معنوي على المتغير المعتمد الممثل بمعدل نمو الناتج المحلي الإجمالي للإقليم. وهذه المتغيرات هي: أ- إجمالي عدد السياح X1 السياح الذين زاروا إقليم كوردستان العراق في نهاية كل سنة من سنوات عينة البحث (2008-2018). ب- إجمالي العوائد السياحية X2، المتغير الثالث والأخير العوائد من السياحة والتي تعتبر المحرك النشط للإقتصاد ككل والقطاع السياحي بالأخص، حسبت وقدرت بعدد السواح مضروية بمقدار ما ينفقونه خلال إقامتهم في الإقليم بالدولار الأميركي. ج- إجمالي فرص العمل المباشرة X3، المتغير الثاني يعبر عن مجموع فرص العمل التي يقدمها القطاع السياحي مباشرة في الإقليم، أي الإثتغال الفعلي في القطاع السياحي، مثل الفنادق ووكالات السفر وشركات النقل وبيع التذاكر والتسويق السياحي والكازينومات ومحل بيع التحف والتذكارات وأماكن اللهو وغيرها.

\section{5}

تم إجراء عدد من الإختبارات لأُنموذج أثر الإستثمار السياحي في تنويع

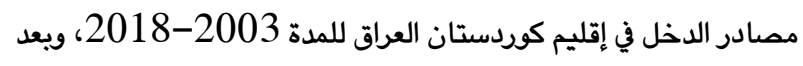
مقارنة نتائج الإختبارات الإحصائية والقياسية ومدى توافقها مع منطوق النظرية الإقتصادية، فقد تمت الإستعانة بالإنموذج النصف اللوغاريتمي الخطي الذاتي في تقدير دالة الإنحدار كونه يعكس طبيعة العلاقة الحقيقية بين المتغيرات المستقلة والمتغير التابع المستخدمين في هذا التحليل وأعطى أفضل التقديرات وظهرت معنوية المتغيرات المتغيرات المستقلة التي إجتازت إختبار (t) الإحصائي. وأن معادلة الإنددار لمعدل النمو في الناتج المحلي الإجمالي لإقليم كوردستان العراق للفترة (20032018) كانت كالآتي: $\mathrm{LnY}=1.93-0.04 \mathrm{X}_{1}+0.001 \mathrm{X}_{2}$ $+0.21 \mathrm{X}_{3}+0.62 \mathrm{LnY}(-1)$
S. E. $=(1.252354)$
$(0.000000105)$
(0.000000000866)
$(0.13)$
$(0.00002)$

T-test (1.545402) (-2.912092)

(4.104655)

$\mathrm{P}$-value (0.1533)

$(0.0155)$

(0.0021)

(0.0009)

$(0.056957)$

F-test $=(15.26233)$

P-value (0.000293)

$\mathrm{R}^{2}=0.859253$

Adjusted R-squared $\left(\mathrm{R}^{-2}\right)=0.802954$

Durbin-Watson stat. $=0.802954$
في تطوير القطاع السياحي، من خلال إقامة المزيد من المنتجعات السياحية ويناء الفنادق الجديدة وإقامة الأماكن الترفيهية وتطويرها، ويناء المدن السياحية وتقديم خدمات أفضل للسياح، أي الإهتمام المتزايد بهذا القطاع الحيوي ودوره في إمتصاص البطالة.

5. قياس أثر الإستثمارات السياحية في الناتج المحلي الإجمالي للإقليم للسنوات (2003-2018)

1.5 توصيف النموذج القياسي:

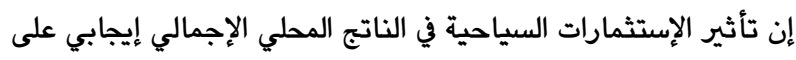
وفق نظرية هكثر أولين في التجارة الدولية، إذ تبين النظرية أن الدولة الإني

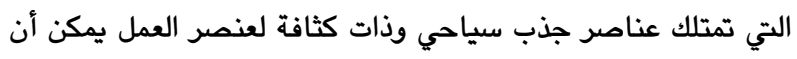

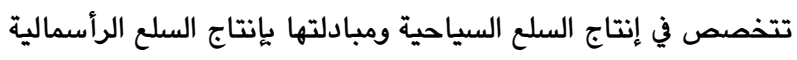

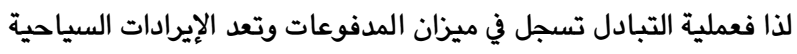

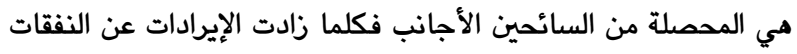
السياحية زاد الأثر الإيجابي في ميزان المدفوعات ويالتالي زيادة الناتج الإنج

$$
\text { المحلي الإجمالي. }
$$

تجدر الإثارة إلى أن إستخدام الأسلوب الإحصائي المعروف بتحليل

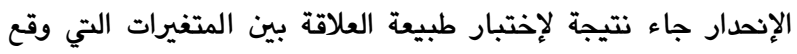

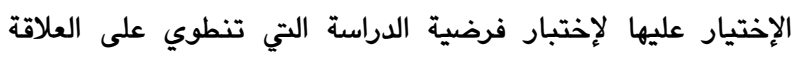

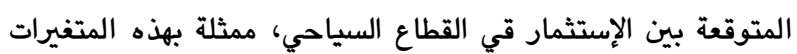

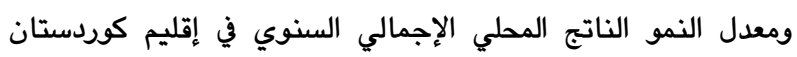

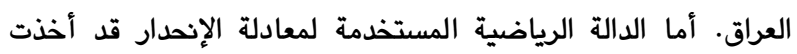
$Y_{i}=F\left(X_{j}\right)$

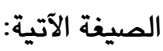

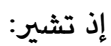
Y إلى معدل نمو الناتج المحلي الإجمالي السنوي لإقليم كوردستان

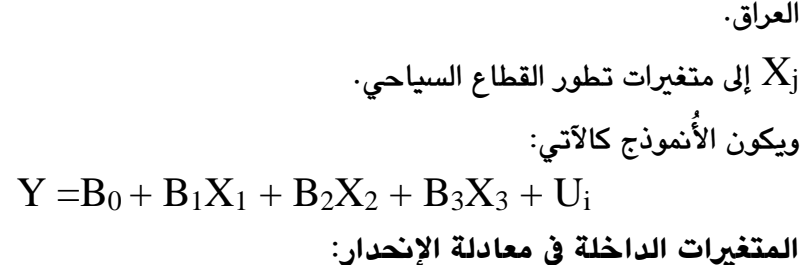
1. المتغير المعتمد: وهو معدل النمو بالناتج الإجمالي المحلي السنوي (GDP) في النموذج، إن أي أي تقدير وتحليل علاقات التأثير

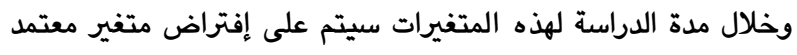

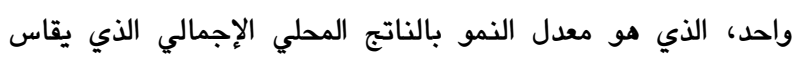
بالأسعار الجارية بالدولار على أساس سعر ثابت للعملة المحلية مقابل الدولار حسب ما إعتمده البنك المركزي العراقي وهو 1170 دينار

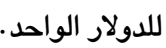

2. المتغيرات المستقلة: وتشير إلى أدوات تطور القطاع السياحي التي وقع الإختيار عليها للاسباب الواردة في أعلاه، التي تفترض الدرات الدراسة التطاع السياحي 
لـجدول (4): أنموذج الإنحدار المقدر للقطاع السياحي في إقليم كوردستان العراق للمدة 2003-2018

\begin{tabular}{|c|c|c|c|c|}
\hline \multicolumn{5}{|c|}{ Dependent Variable: LNY } \\
\hline \multicolumn{5}{|c|}{ Method: Least Squares } \\
\hline \multicolumn{5}{|c|}{ Date: $08 / 24 / 21$ Time: $16: 26$} \\
\hline \multicolumn{5}{|c|}{ Sample (adjusted): 20042018} \\
\hline \multicolumn{5}{|c|}{ Included observations: 15 after adjustments } \\
\hline Variable & Coefficient & Std. Error & t-Statistic & Prob. \\
\hline $\mathrm{C}$ & 1.935391 & 1.252354 & 1.545402 & 0.1533 \\
\hline $\mathrm{X} 1$ & $-3.06 \mathrm{E}-07$ & $1.05 \mathrm{E}-07$ & -2.912092 & 0.0155 \\
\hline $\mathrm{X} 2$ & 4.93E-05 & 0.000866 & 0.056957 & 0.9557 \\
\hline $\mathrm{X} 3$ & $8.20 \mathrm{E}-05$ & $2.00 \mathrm{E}-05$ & 4.104655 & 0.0021 \\
\hline LAGY & 0.629377 & 0.134748 & 4.670754 & 0.0009 \\
\hline R-squared & 0.859253 & \multicolumn{2}{|c|}{ Mean dependent var } & 9.105596 \\
\hline Adjusted R-squared & 0.802954 & \multicolumn{2}{|c|}{ S.D. dependent var } & 0.545075 \\
\hline S.E. of regression & 0.241958 & \multicolumn{2}{|c|}{ Akaike info criterion } & 0.261098 \\
\hline Sum squared resid & 0.585438 & \multicolumn{2}{|c|}{ Schwarz criterion } & 0.497115 \\
\hline Log likelihood & 3.041765 & \multicolumn{2}{|c|}{ Hannan-Quinn criter. } & 0.258584 \\
\hline F-statistic & 15.26233 & \multicolumn{2}{|c|}{ Durbin-Watson stat } & 1.877595 \\
\hline Prob(F-statistic) & 0.000293 & & & \\
\hline
\end{tabular}

المصدر: الجدول من مخرجات الحاسبة الألكترونية.

درجات متباينة من هذا الإرتباط رغم إجتيازها الإختبارات القياسية، والتي ريما يكون لها أثر معنوي على دقة تقديرات قيم بعض المعلمات وهذا من الممكن أن يفسر الإثارات غير المنسجمة مع توقعات النظرية الإقتصادية المسبقة. ويناءاً على المعطيات أعلاه سيتم تقييم النتائج الخاصة بالنموذج وكما يأتي: 1. تشير قيمة معلمة ثابت الإنحدار (B0) الموجبة إلى أنه في غياب المتغيرات المستقلة المعبرة عن التطور في القطاع السياحي، سوف يكون هناك درجة من النمو بالناتج المحلي الإجمالي لإقليم كوردستان العراق. وتبين أن المتغيرات المستقلة معاً لها أثر معنوي أيجابي في نمو الناتج المحلي الإجمالي لسنوات الدراسة وهذا مطابق للمنطوق الإقتصادي، والنظرية الإقتصادية، لكن تفسير ذلك مو لأن إقتصاد الإقليم هو إقتصاد ريعي بإمتياز وتعتبر ضمن الأقاليم مرتفعة الدخل لكن ليس بالضرورة أن يعد قطاعها السياحي متطوراً، كذلك أن قيم المعلمات المقدرة لاتزيد عن الـ (0.6) في النموذج أي أنها تشير إلى إنخفاض

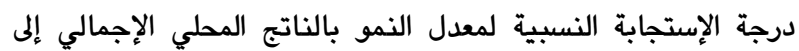
التطور في القطاع السياحي في الإقليم. 2. إن قيم المعلمات (معامل المرونة)، تشير إلى نسبة إستجابة النمو الاقتصادي إلى التغيرات في تلك المتغيرات المستقلة على الرغم من ظهور تأثيرها تأثيراً معنوياً من الناحية الإحصائية.
3.5. تقييم نموذج الإنحدار: نتائج التقديرات وقيم الإختبارات أوحت بإنطباع جيد للنموذج المقدر،

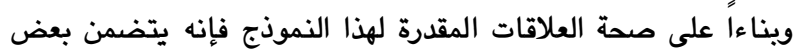
الإستنتاجات الأساسية يستوجب الإثارة اليها في تقييم النتائج وكما

أ. إن درجة الأثر المعنوي للمتغيرات المستقلة كانت أكثر من جيدة وهي إجمالي عدد السياح (X1))، وإجمالي الإيرادات السياحية (X))،

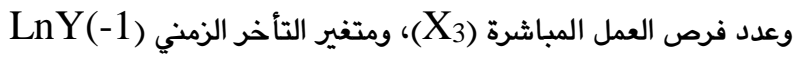
وكانت ضمن حدود الإختبارات الإحصائية. ب. إن حجم المعلمات المقدرة (المرونات) كانت أقل من الواحد الصحيح مما يعطي دلالة على درجة الإستجابة المنخفضة رغم أثرها الإحصائي المعنوي.

ت. لم تظهر وجود حالة الإرتباط الذاتي بين قيم المتغير العشوائي في الأُنموذج المقدر.

ث.إن المتغيرات المستقلة الداخلة في الأُنموذج المقدر جميعها ذات قوة تفسيرية كبيرة للتغيرات في المتغير المعتمد بدلالة أن أقل قيمة لمعامل التحديد المعدل كان (0.802954). ومذا قد يؤشر على درجة جيدة من الدقة في توصيف الأنموذج. ج. يخلو الأنموذج من مشكلة الإرتباط الخطي المتعدد بين المتغيرات المستقلة على وفق الإختبارات القياسية، إلا أن هذا لايعني عدم وجود 


\section{7. المقترحات}

1- إعطاء دور بارذ وأهمية كبيرة للهيئة العامة للسياحة والآثار وعقد إتفاقيات سياحية مع دول الجوار من خلال تسهيل تأشيرات الدخول. 2- توفير بيانات تفصيلية عن السياحة بإقليم كوردستان العراق وإعداد مسوحات دورية لهذا القطاع. 3- تطوير الملاكات البشرية في المرافق السياحية، من خلال إعداد مرشدين سياحيين كفوئين، وتنمية قدراتهم في ميدان الخبرة السياحية، وإختيار من يجيدون التحدث باللغات العربية والإنجليزية، ناهيك عن الكوردية.

4. العمل على تظافر الجهود داخل المجتمع من حكومة (وزارات وهيئات)، ومنظمات من أجل تنمية الوعي المجتمعي بالسياحة وأمميتها الاقتصادية بإستهداف تحسين نوعية السياحة وتطويرها ليإستمرار. 5- إتخاذ الإجراءات اللازمة لصيانة مواقع الآثار وزيادة البحث والتنقيب وحمايتها من أعمال السرقة والتخريب وإصدار قوانين خاصة بها للحد من سرقة الآثار والتجاوز عليها وضرورة ربط هذه المواقع بالطرق المختلفة وتزيينها بلافتات إرثادية وتعريفية خاصة بها. 6- ربط إقليم كوردستان العراق مع دول الجوار بخطوط نقل حديثة وسريعة (خاصة الجوية منها) وسكك حديدية مع إعادة دراسة التشريعات التي تحكم هذه الخدمات. 7- الإعتراف بأهمية القطاع السياحي من لدن الجهات الرسمية ويخاصة الاقتصادية، وتوفير التمويل الإستثماري، والتنسيق مع المؤسسات المالية المختصة لدعم القطاع السياحي وتمويله.

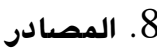

1.8 - 1.8 - المصادر باللغة العربية:

أ) الكتب:

حربي محمد موسى عريقات (2006)، مبادىء الإقتصاد الكلي (التحليل الكلي)، الطبعة الأولى، دار وائل للنشر، عمان-الأردن.

عبدالناصر بن عبدالرحمن وقسيمة الزهراني وكباشي حسين (2008)، الإستثمار

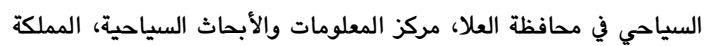

$$
\text { العربية السعودية. }
$$

دريد كامل آل شبيب (2009)، الإستثمار والتحليل الإستثماري، دار اليازوري العلمية للنشر والتوزيع، عمان-الأردن.

ب) الدوريات:

سعيد الحلاق ومريان نادرة (200)، الإستثمار الخاص وأثره على النمو الإقتصادي

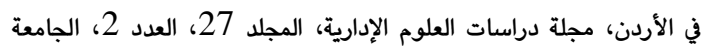

$$
\text { الأردنية، عمان-الأردن. - الاردن. }
$$

نبيل فيصل موسى (2013)، أثر الإستثمار السياحي على التواصل الحضاري للمجتمع، مجلة كلية بغداد للعلوم الإقتصادية الجامعة، العدد 36.
3. تبين أن لمتغير عدد السواح علاقة إرتباط عكسية مع معدلات نمو الناتج المحلي الإجمالي في الإقليم، وهذا في الواقع حالة غير متوقعة من إنمباط الناحية النظرية، إلا أن متابعة طبيعة النشاط الإقتصادي في الإقليم وخاصة خلال مدة الدراسة، قد تعطي مسوغات لهذا السلوك ومن بينها حالات إرتفاع الأسعار (التضخم) في الإقليم نتيجة للتطور الكبير في

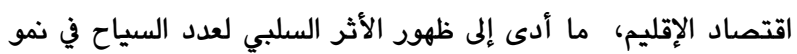
الناتج المحلي الإجمالي، وما ترتب عليه من تردي النشاط السياحي ومن تأثيره في النمو الإقتصادي وإستمرار التباطوء الاقتصادي في الإقليم، ولأن الإقليم يعتمد على وفورات الحجم في السياحة (العدد الكبير) للاسباب الآنفة الذكر فإن العدد القليل من السياح يكون تأثيرما

$$
\text { سلبياً على النمو في الناتج المحلي الإجمالي. }
$$

4. قد يكون مصدر الحجم والشكل الذي ظهرت به قيم المعلمات الخاصة بالمتغيرات الأريع أعلاه عائداً إلى درجة من التداخل الخطي بينها أو عدم دقة البيانات.

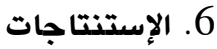

1. تسهم السياحة على مستوى العالم نسبة 10.4٪ من الناتج المحلي الإجمالي العالمي وتستوعب 313 مليون وظيفة، أي نحو 10٪ من إجمالي التوظيف على الصعيد العالمي. ويمعدل نمو سنوي طويل الأجل بلغ أكثر من 5\% للمدة 1950-2018. 2- يتمتع إقليم كوردستان العراق بإمكانات مائلة من موارد طبيعية وحضارية ومقومات سياحية قلما توجد في دولة من دول العالم، وتتميز

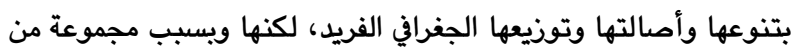
العوائق لم تستثمر بشكل صحيح. 3- من أهم عناصر ديمومة السياحة ونجاحها هو الإستقرار السياسي والأمني ومذا الجانب في الإقليم هش وقابل للتغير في أي لحظة. 4- إغفال كبير من قبل الحكومة للدور الكبير الذي يمكن أن يضطلع به هذا القطاع في تنمية وتحسين الإقتصاد في الإقليم إسوة بباقي دول الجوار مثا تركيا والأردن ومصر والإمارات العربية المتحدة.

5- قلة توافر شركات السياحة في الإقليم وكذلك ضعف دود وزارة البلديات والمصايف والآثار وعدم الإهتمام بالترويج للسياحة في الإقليم. 6- أثبتت الدراسة صحة العلاقة الإيجابية بين الإيراد السياحي والناتج المحلي الإجمالي وهذا يدفع إلى أهمية السياحة كقطاع قائد يمكن أن لن الن يحرك باقي القطاعات. 7- إستطاعت المتغيرات التفسيرية (المستقلة) تفسير 80٪ من التغيرات الحاصلة في المتغير التابع وأن تغير 1\% من إيرادات السياحة تؤدي إلى زيادة الناتج المحلي بمقدار (0.01). 
صالح ، بهرم محمود و الصفار، أحمد سليمان ياسين / مجلة العلوم الانسانية لجامعة زاخو، مجلد:9 ، العدد:3 ، ص 621- 632 ئيلون- 2021.

http://data.albankaldawli.org/indicator/NY.GDP.MKTP. CD.

$$
\text { 2.8 }
$$

Yong Songling, Muhammed Ishtiaq and Bui Thi Thanh, Tourism Industry and Economic Growth Nexus in Bijing China, (2019) School of Management and Economics, Bijing University of Technology, Chaoyang, 100124, Cgina.

Syed Jawad Hussain Shahzad, Muhammad Shahbaz, Roman Ferrer and Ronald Ravinesh Kumar (2017), Tourism-led growth in the top ten tourist destinations: New evidence using the quantile-onquantile approach, Elsevier Journal, Tourism Management 60.

Sinclair, M.T. (1998), Tourism and Economic Development A Survey< Journal of Development Studies, 14 (5), June.

Gujarati, D. N. (2003), Basic Econometrics, $4^{\text {ed }}$, McGraw Hill Gujarati, DN, (2003). Basic Econometrics.

Ivanov, S., \& Webster, C. (2007). Measuring the impact of tourism on economic growth, Tourism Economics, 13(3), 379-388.

http://www.kgptour.tuke.sk/conferences_soubory/pdf

World Tourism organization (2016),UNWTO Annual Madrid. Report 2015,UNWTO,

UNTWO Highlights, 2017 Edition.

UNTWO Highlights, 2018 Edition.

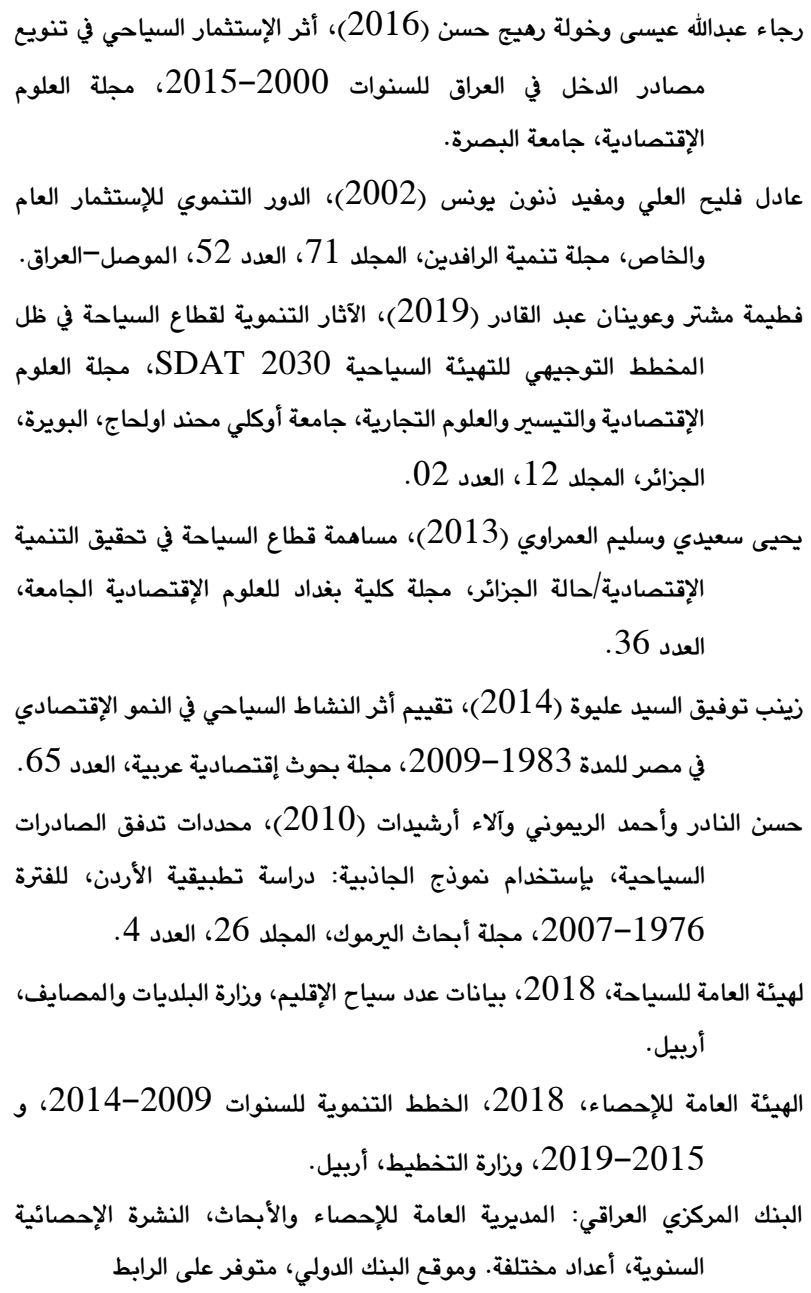


صالح ، بهرم محمود و الصفار، أحمد سليمان ياسين / مجلة العلوم الانسانية لجامعة زاخو، مجلد:9 ، العدد:3 ، ص 621- 632 ئيلون- 2021.

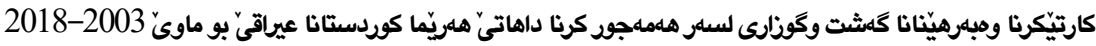

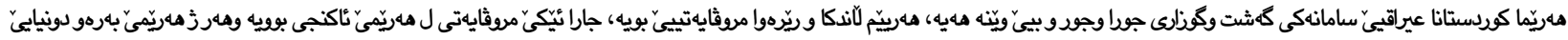

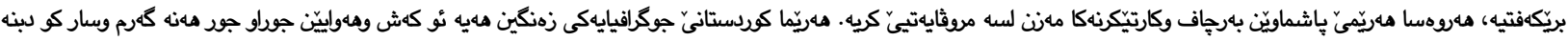

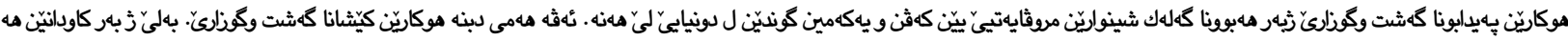

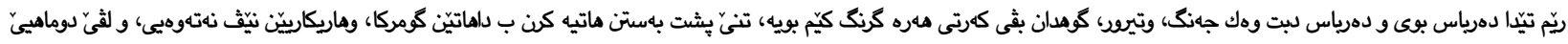

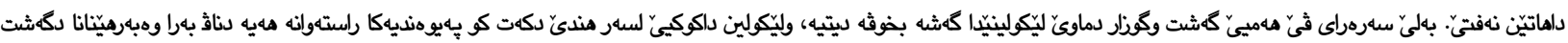

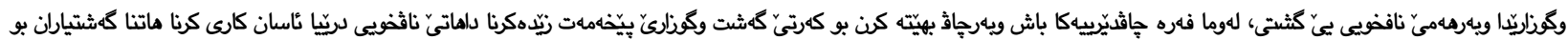

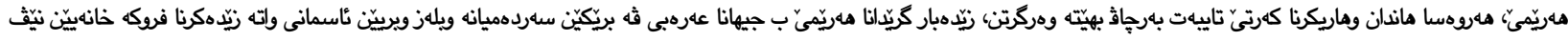

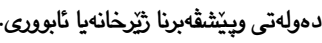

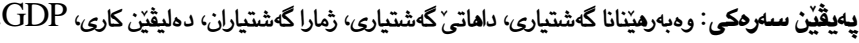

\title{
The impact of tourism investment in diversifying sources of income in the Kurdistan Region of Iraq For the years 2003-2018
}

\begin{abstract}
The Kurdistan Region of Iraq enjoys a diverse and unique tourism wealth. It is the cradle of humanity and the crossing in which the human race settled from Africa and then traveled to the rest of the world. The presence of many traces of the ancient human presence and the patterns of the first human settlements constitute a factor for almost all types of tourism, but due to the conditions that the region in general and Iraq and the Kurdistan Region in particular, wars and suffering over the past five decades, have limited the government's tendency to take care of this sector and the revenues have been approved customs, gifts and international aid, and finally, dependence on oil revenues, but despite everything, tourism remained popular during the study period, and the study adopted a positive impact between tourism investment and GDP, and the study concluded that there is a direct relationship between tourism investment and GDP, so a prominent role must be given And the great importance of tourism in generating income through visa facilities and supporting and paying the private sector more. As well as working to link the region with the Arab world with modern and fast transport lines and airspace, opening the largest possible number of air ports and developing the infrastructure in the region.
\end{abstract}

Keywords: Tourism investment, Tourism revenues, Number of tourists, Job opportunities, Gross domestic product. 